\title{
Profiling of healthy and asthmatic airway smooth muscle cells following interleukin-1ß treatment: a novel role for CCL20 in chronic mucus hypersecretion
}

\author{
Alen Faiz ${ }^{1,2,3,4,5}$, Markus Weckmann ${ }^{1,6}$, Haitatip Tasena ${ }^{3,4,5}$, \\ Corneel J. Vermeulen ${ }^{3,4}$, Maarten Van den Berge ${ }^{3,4}$, Nick H.T. ten Hacken ${ }^{3}$, \\ Andrew J. Halayko ${ }^{7}$, Jeremy P.T. Ward $\mathbb{1}^{8}$, Tak H. Lee ${ }^{8}$, Gavin Tjin ${ }^{1,2,9}$, \\ Judith L. Black ${ }^{1,2,9}$, Mehra Haghi ${ }^{10}$, Cheng-Jian Xu ${ }^{4,11}$, Gregory G. King ${ }^{1,2,12}$, \\ Claude S. Farah ${ }^{1,2,13}$, Brian G. Oliver ${ }^{1,14}$, Irene H. Heijink ${ }^{3,4,5}$ and \\ Janette K. Burgess ${ }^{1,2,4,9,5}$
}

@ERSpublications

Elevated levels of CCL20 contribute to enhanced mucus hypersecretion in asthma http://ow.ly/m7F830ko3Rb

Cite this article as: Faiz A, Weckmann $\mathrm{M}$, Tasena $\mathrm{H}$, et al. Profiling of healthy and asthmatic airway smooth muscle cells following interleukin-1 $\beta$ treatment: a novel role for CCL20 in chronic mucus hypersecretion. Eur Respir J 2018; 52: 1800310 [https://doi.org/10.1183/13993003.00310-2018].

ABSTRACT Chronic mucus hypersecretion ( $\mathrm{CMH})$ contributes to the morbidity and mortality of asthma, and remains uncontrolled by current therapies in the subset of patients with severe, steroidresistant disease. Altered cross-talk between airway epithelium and airway smooth muscle cells (ASMCs), driven by pro-inflammatory cytokines such as interleukin (IL)-1 $\beta$, provides a potential mechanism that influences $\mathrm{CMH}$. This study investigated mechanisms underlying $\mathrm{CMH}$ by comparing IL- $1 \beta$-induced gene expression profiles between asthma and control-derived ASMCs and the subsequent paracrine influence on airway epithelial mucus production in vitro.

IL-1 $\beta$-treated ASMCs from asthmatic patients and healthy donors were profiled using microarray analysis and ELISA. Air-liquid interface (ALI)-cultured CALU-3 and primary airway epithelial cells were treated with identified candidates and mucus production assessed.

The IL-1 $\beta$-induced CCL20 expression and protein release was increased in ASMCs from moderate compared with mild asthmatic patients and healthy controls. IL-1 $\beta$ induced lower MIR146A expression in asthma-derived ASMCs compared with controls. Decreased MIR146A expression was validated in vivo in bronchial biopsies from 16 asthmatic patients versus 39 healthy donors. miR-146a-5p overexpression abrogated CCL20 release in ASMCs. CCL20 treatment of ALI-cultured CALU-3 and primary airway epithelial cells induced mucus production, while CCL20 levels in sputum were associated with increased levels of $\mathrm{CMH}$ in asthmatic patients.

Elevated CCL20 production by ASMCs, possibly resulting from dysregulated expression of the antiinflammatory miR-146a-5p, may contribute to enhanced mucus production in asthma. 
Affiliations: ${ }^{1}$ Woolcock Institute of Medical Research, The University of Sydney, Glebe, Australia. ${ }^{2}$ Sydney Medical School, The University of Sydney, Sydney, Australia. ${ }^{3}$ Dept of Pulmonary Diseases, University of Groningen, University Medical Center Groningen, Groningen, The Netherlands. ${ }^{4}$ GRIAC IGroningen Research Institute for Asthma and COPD), University of Groningen, University Medical Center Groningen, Groningen, The Netherlands. ${ }^{5}$ Dept of Pathology and Medical Biology, University of Groningen, University Medical Center Groningen, Groningen, The Netherlands. 'Section for Pediatric Pneumology and Allergology, University Medical Center Schleswig-Holstein, Campus Centrum Luebeck, Airway Research Centre North (ARCN), Member of the German Centre of Lung Research (DZL), Luebeck, Germany. ${ }^{7}$ University of Manitoba/Manitoba Institute of Child Health - Winnipeg, Winnipeg, MB, Canada. ${ }^{8}$ Dept of Physiology, Kings College London, London, UK. ${ }^{9}$ Discipline of Pharmacology, Faculty of Medicine, The University of Sydney, Sydney, Australia. ${ }^{10}$ Graduate School of Health, University of Technology Sydney, Sydney, Australia. ${ }^{11}$ Dept of Pediatric Pulmonology and Pediatric Allergology, Beatrix Children's Hospital, University Medical Center Groningen, Groningen, The Netherlands. ${ }^{12}$ Dept of Respiratory Medicine, Royal North Shore Hospital, St Leonards, Australia. ${ }^{13}$ Dept of Respiratory Medicine, Concord Hospital, Concord, Australia. ${ }^{14}$ School of Medical and Molecular Biosciences, University of Technology Sydney, Sydney, Australia.

Correspondence: Alen Faiz, Dept of Pathology and Medical Biology, University Medical Center Groningen, Hanzeplein 1, 9713 GZ Groningen, The Netherlands. E-mail: a.faizQumcg.nl

\section{Introduction}

Asthma is a chronic inflammatory disease affecting 300 million people worldwide [1]. Chronic mucus hypersecretion $(\mathrm{CMH})$ contributes to the morbidity and mortality of asthma [2], and remains uncontrolled by current therapies. There is an urgent need to identify new therapeutic targets.

Although mucus production increases in the airway epithelial layer during inflammation [3], the underlying mechanism of $\mathrm{CMH}$ remains to be elucidated. Differentiation of airway epithelial cells into either ciliated or goblet cells is directed by other structural cells in the submucosa. The cross-talk between the epithelial layer and airway smooth muscle cells (ASMCs) may regulate mucus production, since the airway smooth muscle mass is enlarged in asthma [4].

ASMCs have long been thought to have a passive role, but accumulating evidence suggests that these cells play an important role in the inflammatory process that underlies $\mathrm{CMH}$, providing an active source of cytokines and chemokines via a number of pathways [5].

One of these inflammatory pathways know to be altered in asthma is the inflammasome, a multiprotein complex that plays an important role in the activation of pro-inflammatory cytokines, e.g. conversion of interleukin (IL)- $1 \beta$ from its pro-form into its active state [6]. The activity of the inflammasome is enhanced in neutrophilic asthma [7], leading to increased levels of active IL-1 $\beta$ in sputum.

IL-1 $\beta$ is a strong pro-inflammatory signalling molecule, the downstream mediators of which are associated with mucus production [8]. However, little is known about the influence of IL-1 $\beta$ on the pro-inflammatory response of airway structural cells, especially ASMCs and the potential role in CMH in asthma.

In this study we identified CCL20 and MIR146A when comparing gene expression profiles between asthmatic and healthy ASMCs in vitro in response to IL-1 $\beta$. Importantly, CCL20 had been shown to induce MUC5AC expression in epithelial cultures by binding to its only known receptor CCR6 [9]. Furthermore, in murine models, anti-CCL20 treatment significantly decreased virus-induced mucus production [10]. Previously, a single nucleotide polymorphism (SNP) in miR-146a has been associated with the presence of asthma and other pro-inflammatory diseases [11, 12]. Interestingly, we have recently shown that lower expression of miR-146a-5p in bronchial biopsies is inversely correlated with $\mathrm{CMH}$ in chronic obstructive pulmonary disease (COPD) [13], highlighting miR-146a-5p as a regulator of mucus regulation in respiratory diseases. Based on known roles of CCL20 and miR-146a-5p in mucus production, we then investigated how these factors produced by ASMCs influence mucus production in airway epithelial cells.

\section{Methods}

\section{Human tissue}

Primary human ASMCs were obtained as described previously $[14,15]$ with ethical approval from The University of Sydney and participating hospitals (Concord Repatriation General Hospital, Sydney South West Area Health Service and Royal Price Alfred Hospital) in Sydney, Australia. All patients, or next of kin, provided written informed consent. An outline of the patients' characteristics is shown in table 1.

\section{Microarray processing and analysis}

ASMCs were isolated from asthmatic patients $(n=3)$ and healthy controls $(n=3)$, and cultured as previously described (Dataset A) [14, 15]. Cells were treated with $10 \mathrm{ng} \cdot \mathrm{mL}^{-1} \mathrm{IL}-1 \beta$ (R\&D Systems, Minneapolis, 
TABLE 1 Demographics of individual patients from whom samples were obtained.

\begin{tabular}{|c|c|c|c|c|c|c|}
\hline Patient & Diagnosis & Age years & Sex & Samples & FEV $1 \%$ pred & $\begin{array}{l}\text { Experiment(s) for which } \\
\text { sample was used }\end{array}$ \\
\hline 1 & Asthma & 33 & Male & Bronchoscopy & NA & 1 \\
\hline 3 & Asthma & 33 & Female & Transplant & NA & 1 \\
\hline 4 & Nondiseased donor & 31 & Male & Bronchoscopy & NA & 1 \\
\hline 5 & Nondiseased donor & 22 & Male & Bronchoscopy & NA & 1 \\
\hline 8 & Unknown & NA & Female & Transplant & NA & 2 \\
\hline 9 & Asthma & 19 & Female & Bronchoscopy & 97 & 2 \\
\hline 10 & Nondiseased donor & 20 & Male & Bronchoscopy & NA & 2 \\
\hline 11 & Nondiseased donor & 30 & Male & Bronchoscopy & NA & 2 \\
\hline 12 & Nondiseased donor & 21 & Female & Bronchoscopy & NA & 2 \\
\hline 13 & Nondiseased donor & 31 & Female & Immortalised ASMCs & 105 & 3,4 \\
\hline 19 & Asthma & 21 & Male & Immortalised ASMCs & 108 & 3,4 \\
\hline 20 & Asthma & 31 & Male & Immortalised ASMCs & 85 & 3,4 \\
\hline 21 & Asthma & 27 & Female & Immortalised ASMCs & 78 & 3,4 \\
\hline 22 & Asthma & 33 & Male & Immortalised ASMCs & 78 & 3,4 \\
\hline 23 & Nondiseased donor & 69 & Male & Immortalised ASMCs & NA & 4 \\
\hline 24 & Nondiseased donor & 22 & Female & Immortalised ASMCs & NA & 4 \\
\hline 25 & Nondiseased donor & NA & NA & Immortalised ASMCs & NA & 6 \\
\hline 26 & Cystic fibrosis & 22 & Female & Paraffin-embedded bronchus & NA & 5 \\
\hline 27 & Nondiseased donor & 16 & Male & Paraffin-embedded bronchus & NA & 5 \\
\hline 28 & COPD & 56 & Female & Paraffin-embedded bronchus & NA & 5 \\
\hline 29 & Pulmonary fibrosis & 53 & Female & Paraffin-embedded bronchus & NA & 5 \\
\hline
\end{tabular}

FEV1: forced expiratory volume in $1 \mathrm{~s}$; ASMC: airway smooth muscle cell; NA: not available; COPD: chronic obstructive pulmonary disease. ${ }^{\#}$ : 1 , Human Gene 1.0 ST microarray (Dataset A); 2, Human U133Plus 2.0 microarray (Dataset B); 3, quantitative real-time PCR validation; 4, CCL20 ELISA; 5, CCR6 immunohistochemistry; 6, miR-146a-5p functional work.

MN, USA) for $8 \mathrm{~h}$ [16]. Total cellular mRNA was isolated, labelled and run on a GeneChip Human Gene 1.0 ST Array (Affymetrix, Santa Clara, CA, USA) according to the manufacturer's instructions (Gene Expression Omnibus identifier GSE63383) [4]. For independent validation, ASMCs derived from asthmatic patients $(n=2)$ and healthy donors $(n=4)$ were grown and treated with IL-1 $\beta$ in the same manner (Dataset B). Samples were labelled and run on a Human U133Plus 2.0 Array (Affymetrix) according to the manufacturer's instructions. Microarray analysis is outlined in the supplementary material.

\section{Pathway analysis}

Functional enrichment analysis to identify the overlapping genes altered by IL-1 $\beta$ treatment in Datasets A and B was performed using gene set enrichment analysis (GSEA) software version 2.0.14 (http://software. broadinstitute.org/gsea). Protein network analysis was conducted using STRING version 10 (https:// string-db.org) on the overlapping genes. GSEA was also used to investigate pathways regulated in Dataset A using the BioCarta database (www.biocarta.com). Transcript factor binding site analysis was conducted using g:Profiler (https://biit.cs.ut.ee/gprofiler).

\section{Microarray candidate validation}

The validation of the microarray results was undertaken at a transcriptional (quantitative real-time PCR) and a translational (ELISA) level as described in the supplementary material.

\section{Bronchial biopsies processing for quantification of MIR146A expression}

Bronchial biopsies were collected from respiratory healthy subjects [17] and current asthma patients [18, 19] with a previous doctor's diagnosis of asthma, documented reversibility and airway hyperresponsiveness to histamine (provocative dose causing a $20 \%$ fall in forced expiratory volume in $1 \mathrm{~s}$ for histamine (using $30-\mathrm{s}$ 
tidal breathing) $<32 \mathrm{mg} \cdot \mathrm{mL}^{-1}$ ). The analysis was conducted on 39 healthy subjects and 16 asthmatic patients, who were all nonsmokers and currently not taking inhaled corticosteroids. An outline of the patients' characteristics is shown in supplementary table S1. All study protocols were approved by the medical ethics committee of the University Medical Center Groningen (Groningen, The Netherlands) and all subjects provided written informed consent. RNA was isolated and sequenced as described in the supplementary material.

miR-146a-5p predicted targets

To identify downstream targets of miR-146a-5p, we used a publically available microarray dataset (Gene Expression Omnibus identifier GSE79340) of human hepatic Huh7.5.1 cells transfected with a miR-146a-5p mimic ( $5 \mathrm{nM})$ compared with a negative control $(\mathrm{n}=3)$.

\section{Functional analysis}

Immortalised ASMCs were grown to $80-90 \%$ confluence and serum deprived before transfection with either a miR-146a-5p (100 nM) mimic or mimic control using RNAiMAX (Invitrogen, Carlsbad, CA, USA). $24 \mathrm{~h}$ later, cells were treated with $10 \mathrm{ng} \cdot \mathrm{mL}^{-1} \mathrm{IL}-1 \beta$ or $0.1 \%$ bovine serum albumin (control). Cell-free supernatants were collected at $24 \mathrm{~h}$ and IL- 8 levels assessed by ELISA.

The human lung epithelial cell line CALU-3 and primary airway epithelial cells were grown at the airliquid interface (ALI), treated with $10 \mathrm{ng} \cdot \mathrm{mL}^{-1}$ CCL20 for $48 \mathrm{~h}$ and mucus assessed by Alcian blue staining as described in the supplementary material.

\section{CCL20 levels in sputum}

Sputum was induced in a population of asthmatic patients $(n=89)$, as previously described [20,21], with and without CMH. CCL20 was measured by ELISA.

\section{Definition of $\mathrm{CMH}$}

To define $\mathrm{CMH}$, asthmatic patients were asked to respond to a clinical questionnaire: "How often did you cough up sputum during the last week?" [22]. This question had seven possible answers: 1) never, 2) sometimes, 3) once in a while, 4) often, 5) most of the time, 6) regularly and 7) always. Patients who responded 1) were classified as no $\mathrm{CMH}$, patients who responded 2) and 3) were classified as moderate $\mathrm{CMH}$, and patients who responded 4)-7) were classified as severe $\mathrm{CMH}$. Of the asthmatic patients with available sputum, $\mathrm{n}=80$ gave answers to the questionnaire and were analysed in this study.

\section{Statistics}

Statistical tests and graph plotting were conducted using Prism version 6 (GraphPad, La Jolla, CA, USA). A p-value $<0.05$ was considered statistically significant.

\section{Results}

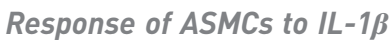

To evaluate if IL-1 $\beta$ is involved in the abnormal cross-talk between ASMCs and airway epithelium, contributing to $\mathrm{CMH}$ in the asthmatic airway, we first examined the regulation of genes following IL- $1 \beta$ treatment. Asthmatic patients $(n=3)$ and controls $(n=3)$ were pooled to obtain sufficient power to determine the effect of IL-1 $\beta$ on gene expression (Dataset A). Gene expression analysis identified 408 genes that were upregulated and 143 genes that were downregulated upon IL-1 $\beta$ treatment compared with baseline (fold change $> \pm 2$, false discovery rate (FDR) $<0.05$ ) (supplementary table S2). Figure $1 \mathrm{a}$ and $\mathrm{b}$ illustrate the genes significantly altered by IL-1 $\beta$ and a volcano plot, respectively.

To validate these findings, we investigated a second independent dataset from $n=2$ asthmatic and $n=4$ healthy-derived ASMC cultures treated with $10 \mathrm{ng} \cdot \mathrm{mL}^{-1} \mathrm{IL}-1 \beta$ for $8 \mathrm{~h}$ (Dataset B). Gene expression analysis identified 377 genes that were upregulated and 98 genes that were downregulated during IL-1 $\beta$ treatment compared with baseline (fold change $> \pm 2$, FDR $<0.05$ ). GSEA of the two datasets revealed that upon IL-1 $\beta$ treatment, 255 of the significantly upregulated genes and 111 of the significantly downregulated genes were core-enriched in the same direction in the two datasets (figure 1c).

Pathway analysis of Dataset A revealed that the majority of pathways increased by IL-1 $\beta$ were pro-inflammatory, including the NF- $\mathrm{BB}$, IL-1 receptor (IL-1R) and TID (chaperones that modulate interferon (IFN) signalling pathway) pathways (figure 1d). Protein network analysis (using STRING version 10.0) showed that the increased IL-1 $\beta$ expression signature in ASMCs was enriched for proteinprotein interactions, indicating that the identified genes may have similar functions (figure 1e). Three distinct clusters were identified: IFN-related genes, NF- $\kappa \mathrm{B}$ signalling-related genes and pro-inflammatory cytokines. NF- $\mathrm{KB}$ and IFN regulatory factor 1 (IRF1) were identified as central hub proteins connecting a 

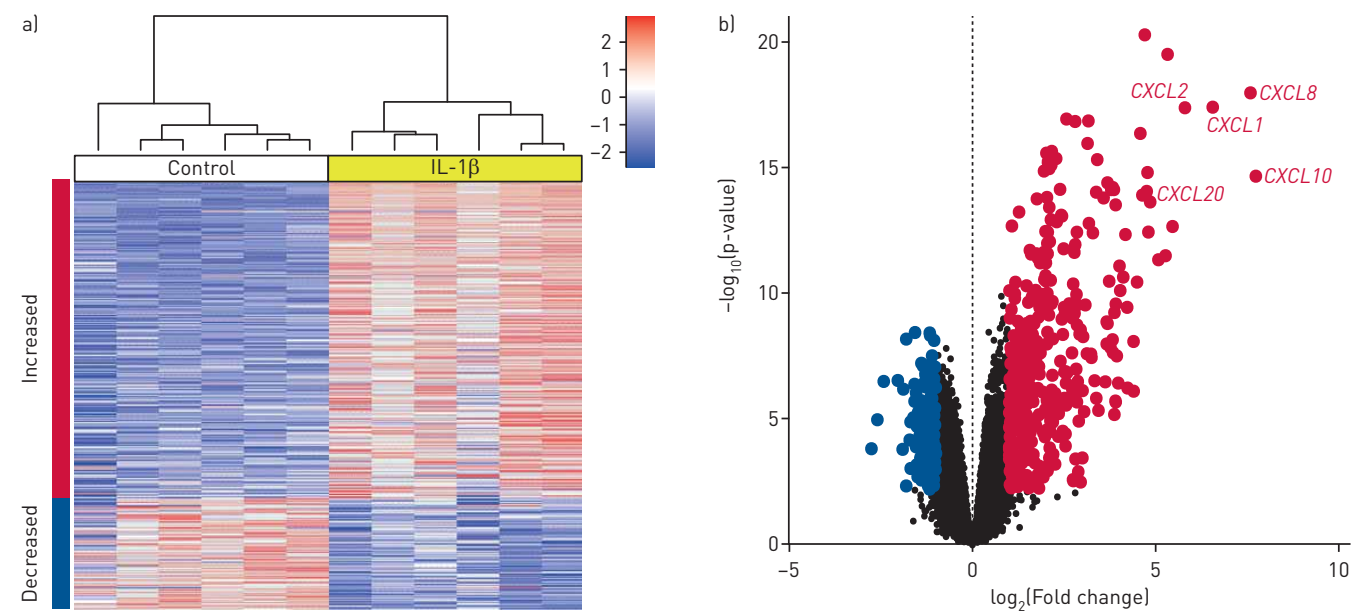

c)

Genes downregulated by IL-1 $\beta$ (Dataset B)

d)

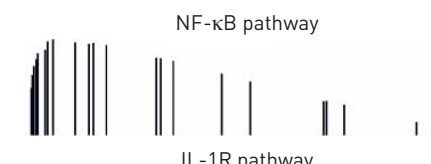

Genes downregulated by IL-1 (Dataset A)

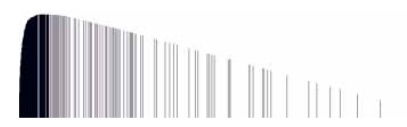

Genes
increased

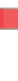

Gene expression in ASMCs

Genes

(Dataset B)

by IL-1 $\beta$

(Dataset B)
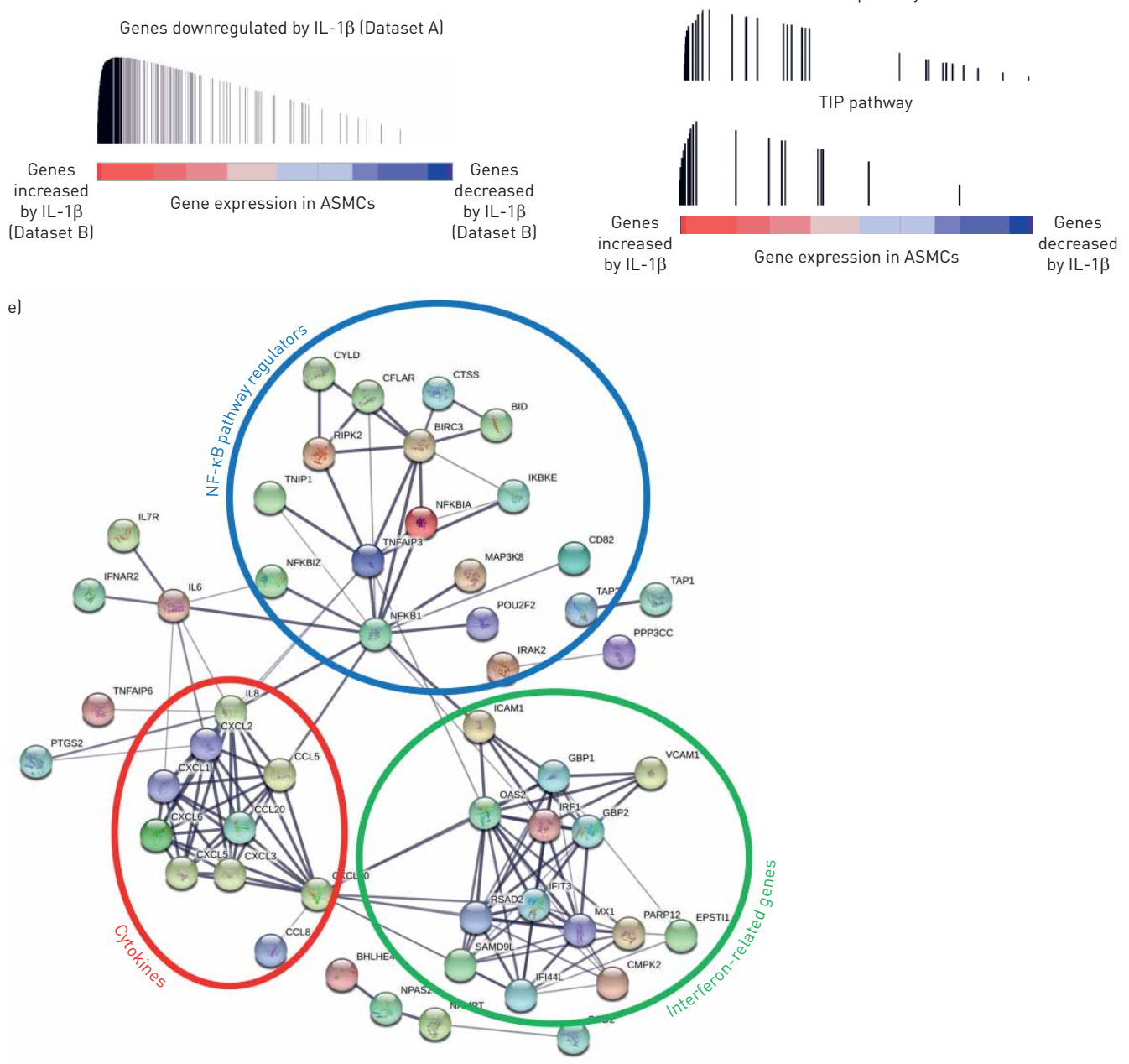

FIGURE 1 Treatment of airway smooth muscle cells (ASMCs) with interleukin (IL)-1 $\beta$. IL-1R: IL-1 receptor; GSEA: gene set enrichment analysis; FDR: false discovery rate. a) Heatmap and b) volcano plot of genes altered by IL- $1 \beta$ treatment for $8 \mathrm{~h}$ in ASMCs (fold change $> \pm 2$, FDR $<0.05$ ). c) GSEA: enrichment of genes upregulated and downregulated by IL-1 $\beta$ treatment in ASMCs comparing two independent datasets (GSEA FDR <0.05). d) GSEA: enrichment of genes involved in the NF- $\kappa B, I L-1 R$ and TID pathways with genes upregulated by IL-1 $\beta$ treatment in ASMCs (GSEA FDR <0.05). Coloured bars in (c) and (d) represent genes ranked based on their differential expression to treatment in ASMCs; vertical bars represent the running GSEA enrichment score and location (in the ranked gene list) of genes that are involved in the pathway being tested. e) Protein interaction analysis. 
number of protein network clusters together, indicating a central role of these proteins during IL-1 $\beta$ stimulation in ASMCs (figure 1e). Six of the top 10 genes upregulated by IL-1 $\beta$ formed a clear individual cluster (cytokines), which included the CXCL family proteins CXCL8 (a cytokine previously associated with neutrophilic airway inflammation in asthma [23]), CXCL10 (an IFN-regulated cytokine associated with mast cell migration [24]) and CCL20 (a chemoattractant for CCR6 ${ }^{+}$immature dendritic cells, T-helper 17 cells and neutrophils).

Transcription factor binding analysis conducted on the overlapping genes between Datasets A and B using g:Profiler identified that the upregulated genes were enriched for NF- $\kappa B$ (FDR $7.84 \times 10^{-10}$ ), RelA (a component of the NF- $\mathrm{KB}$ complex; FDR $7.18 \times 10^{-13}$ ) and IRF1 (FDR $1.72 \times 10^{-13}$ ) transcription factor binding sites, while the downregulated genes were enriched for ETF (FDR $5.57 \times 10^{-7}$ ) and EGR1 (early growth response 1; FDR $5.64 \times 10^{-5}$ ) transcription factor binding sites. These results again identify NF- $\kappa \mathrm{B}$ and IRF1 as key regulators of IL-1 $\beta$ signalling in ASMCs.

Importantly, CCL20 has been shown to induce MUC5AC expression in epithelial cultures [10, 25]. Furthermore, in murine models, anti-CCL20 treatment significantly decreased virus-induced mucus production. Based on its known role in mucus production, we selected CCL20 for further functional studies.

\section{CCL20 protein release induced by IL-1 $\beta$ in ASMCs}

To validate the microarray findings, CCL20 mRNA expression was measured following the stimulation of the asthmatic (mild and moderate) and healthy-derived ASMCs from Dataset A with $10 \mathrm{ng} \cdot \mathrm{mL}^{-1} \mathrm{IL}-1 \beta$ for $8 \mathrm{~h}$. IL-1 $\beta$ significantly increased CCL20 expression, supporting the microarray results (figure 2a). No differences were found in mRNA expression between asthmatic and healthy-derived ASMCs or between ASMCs from mild and moderate asthmatic patients. Next, we confirmed our findings at the protein level and observed that IL-1 $\beta$ significantly increased CCL20 release from ASMCs after $24 \mathrm{~h}$ (figure 2b). The levels of CCL20 were more strongly elevated in moderate asthmatic ASMCs compared with those from both healthy controls and mild asthmatic patients. Levels of CCL20 released from ASMCs at baseline were equivalent to levels previously reported to be released by epithelial cells [20].

MIR146A is decreased in asthma and regulates CCL20

Having seen that CCL20 protein was differentially regulated by IL-1 $\beta$ in asthmatic compared with healthy-derived ASMCs, we investigated other IL-1 $\beta$-induced genes.

This analysis was conducted on a subset of genes regulated by IL-1 $\beta$ treatment in Dataset A (fold change $> \pm 2$, FDR <0.05). Only a single transcript, MIR146A, had a significantly smaller increase in gene expression upon IL-1 $\beta$ treatment in asthmatic-derived ASMCs compared with ASMCs from healthy controls (fold change $> \pm 2$, FDR $<0.05$ ) (figure $3 \mathrm{a}$ ). MIR146A is the precursor transcript for miR-146a-3p
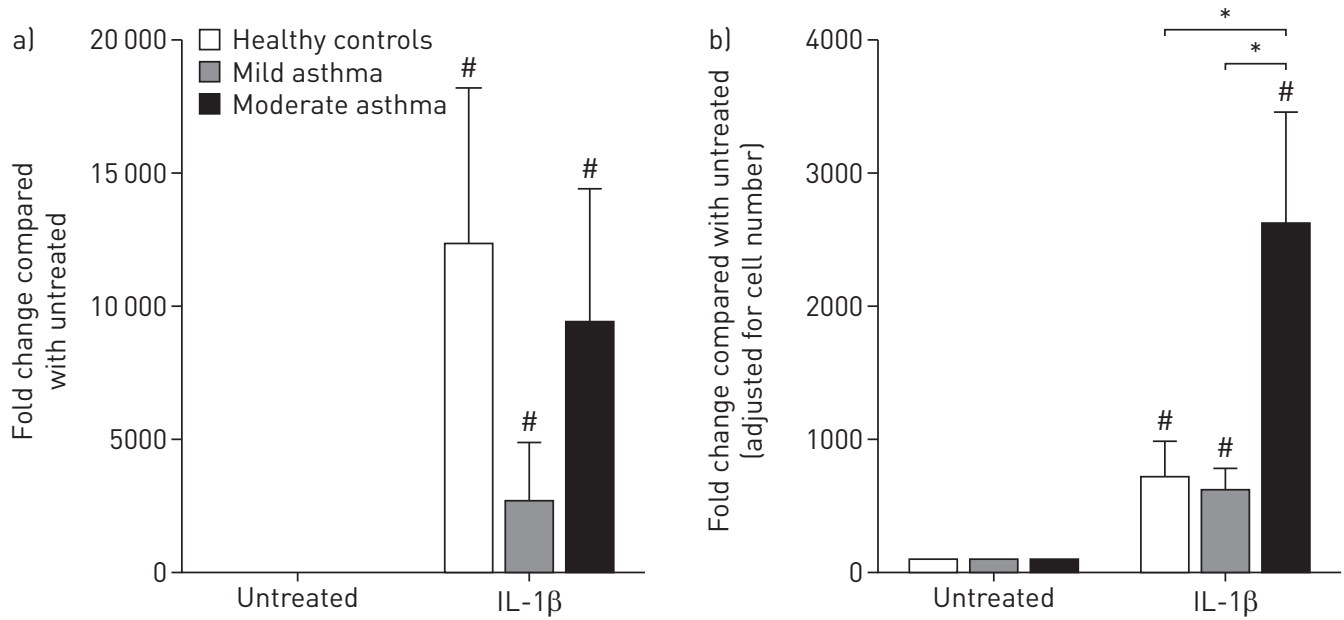

FIGURE 2 Interleukin (IL)-1 $\beta$ induced production of CCL20 mRNA by airway smooth muscle cells (ASMCs). ASMCs were grown to confluence in growth media, quiesced for $72 \mathrm{~h}$ and then treated with $0.1 \%$ bovine serum albumin (control) or $10 \mathrm{ng} \cdot \mathrm{mL}^{-1} \mathrm{IL}-1 \beta$ for $8 \mathrm{~h}$ (mRNA) or $24 \mathrm{~h}$ (protein). a) mRNA levels of CCL20 (healthy controls $n=4$, mild asthma $n=3$ and moderate asthma $n=3$ ). b) CCL20 protein levels were measured in cell-free supernatant (healthy controls $n=4$, mild asthma $n=3$ and moderate asthma $n=3$ ). Data are presented as mean \pm SEM. Statistical analysis was performed using the paired t-test and t-test for paired and unpaired samples, respectively. ${ }^{*}$ : $p<0.05$, compared with healthy controls; ${ }^{\#}: p<0.05$, compared with IL- $1 \beta$ treatment. 

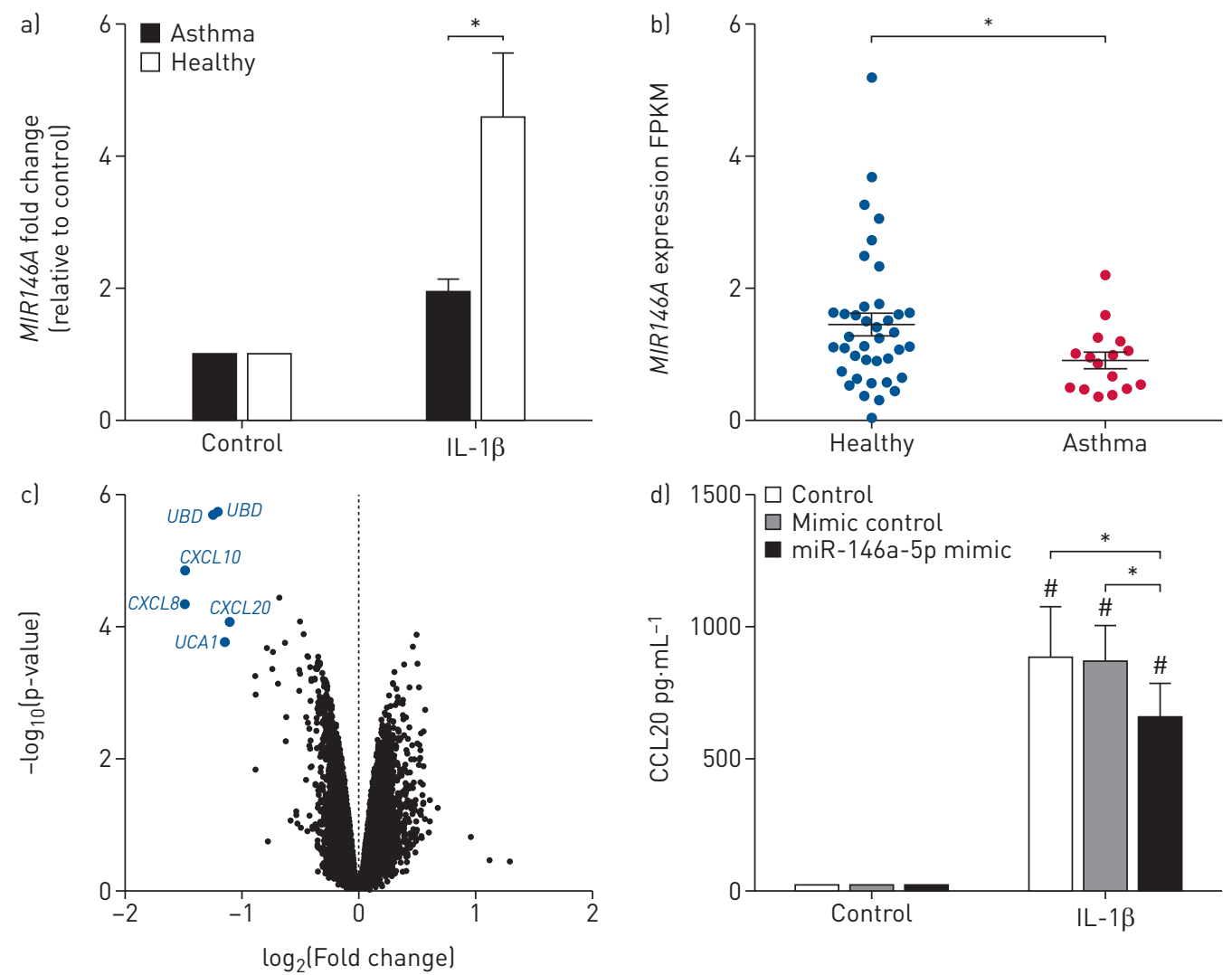

FIGURE 3 Function of miR-146a-5p in airway smooth muscle cells (ASMCs). IL: interleukin; FPKM: fragments per kilobase of transcript per million mapped reads. a) Microarray results of ASMCs treated with IL-1 $\beta$ for $8 \mathrm{~h}$ from asthmatic patients ( $n=3)$ and healthy controls $(n=3)$. b) MIR146A expression in bronchial biopsies from asthmatic patients and healthy controls. c) Volcano plot of Huh7.5.1 cells transfected with a miR-146a-5p mimic $(5 \mathrm{nM})$ compared with a negative control $(n=3)$. d) CCL20 levels from ASMCs treated with IL-1 $\beta$ in the presence and absence of miR-146a-5p (mimic) overexpression compared with a mimic control $(n=5)$. Data are presented as mean \pm SEM. Statistical analysis was performed using the paired t-test and t-test for paired and unpaired samples, respectively. ${ }^{*}: p<0.05 ;{ }^{\#}: p<0.05$, treatment compared with control.

and miR-146a-5p, the latter being a well-known anti-inflammatory microRNA (miRNA), identified to be dysregulated in a number of inflammatory diseases [26]. To determine whether MIR146A expression was altered in asthmatic patients in vivo, we investigated its expression in bronchial biopsies from 16 asthmatic patients and 39 healthy controls. MIR146A expression was decreased in asthmatic bronchial biopsies compared with healthy controls, reflecting the in vitro results (figure $3 \mathrm{~b}$ ).

To identify the function of MIR146A, we focused on the known anti-inflammatory mature transcript miR-146a-5p, and studied direct and indirect targets of this transcript using a publically available dataset of gene expression in Huh7.5.1 cells overexpressing miR-146a-5p. Gene expression analysis identified five genes (UBD (ubiquitin D), CXCL10, CXCL8, CCL20 and UCA1 (urothelial cancer associated 1)) that were downregulated, but no genes that were upregulated upon miR-146a-5p overexpression (fold change $> \pm 2$, FDR <0.05). A volcano plot is shown in figure $3 \mathrm{c}$ and a table of significant genes is given in supplementary table S3. One of the downregulated genes, CCL20, is known to be modulated by miR-146a-5p [27]. Therefore, we investigated whether miR-146a-5p negatively regulates IL-1 $\beta$-induced CCL20 protein release in ASMCs. Overexpression of the miR-146a-5p mimic in immortalised ASMCs led to a significant downregulation of IL-1 $\beta$-induced CCL20 release (figure $3 \mathrm{~d}$ ).

\section{CCL20 receptor CCR6 is present on structural cells of the airways and CCL20 induces mucus production by CALU-3 cells grown at the ALI}

Having identified CCL20 as a mediator that is differentially expressed in asthmatic compared with healthy-derived ASMCs, we next wanted to understand the functional consequences of increased CCL20 in the asthmatic airways. First, to determine whether structural cells in the airways are able to respond to CCL20, the expression of CCR6, its unique receptor, was investigated in airway cells. Initially, we 
performed real-time PCR in human primary ASMCs, immortalised ASMCs and CALU-3 cells, which showed detectable levels of CCR6 mRNA (supplementary figure S1). Immunohistochemistry staining for CCR6 in human bronchial sections confirmed expression on both ASMCs and airway epithelium (supplementary figure S2).

Previously, in murine models, anti-CCL20 treatment significantly decreased mucus production in response to respiratory syncytial virus infection and CCL20 has been shown to induce MUC5AC expression in submerged culture $[9,10]$. To determine whether CCL20 can directly promote mucus production in a model of differentiated epithelial cells, CALU-3 cells were grown at the ALI and allowed to differentiate into mucus-producing cells before being treated basolaterally with physiologically relevant levels of CCL20. CCL20 treatment of CALU-3 cells for $48 \mathrm{~h}$ increased the production of mucus measured by Alcian blue staining (figure 4a). CCL20-induced mucus production was significantly reduced with the specific anti-CCL20 antibody in CALU-3 cells, while the isotype control had no significant effect (figure $4 \mathrm{~b}$ ). A trend in the same direction was found by Alcian blue staining upon CCL20 treatment of ALI-differentiated primary human airway epithelial cultures $(p=0.0625)$ (figure $4 c-e)$. MUC5AC protein levels measured in ALI apical wash were also found to be increased by CCL20 treatment (figure 4f).

\section{Sputum levels of CCL20 are associated with mucus hypersecretion in asthma}

Previous studies have shown that mesenchymal factors can cross the basal lamina propria and be found in lung fluids [28]. This phenomenon is thought to be enhanced in asthmatic patients due to the documented leaky nature of the epithelial layer [29], allowing trafficking to the mucosal layer, where it may induce mucus production. To determine whether CCL20 levels are associated with mucus production in asthmatic patients, we investigated sputum levels of CCL20 in asthmatic patients with and without $\mathrm{CMH}$. Of the 80 patients included, 24 were classified as having no $\mathrm{CMH}, 32$ were classified as having mild

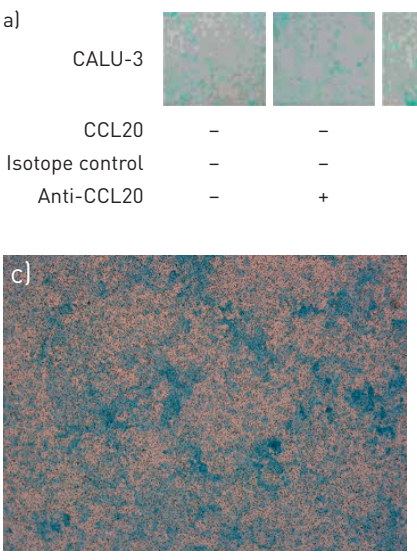

Control

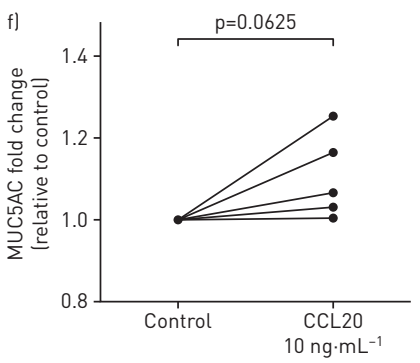

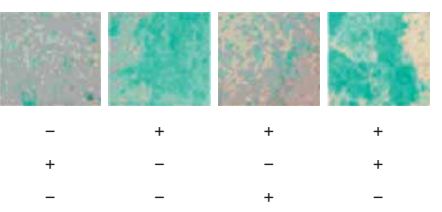

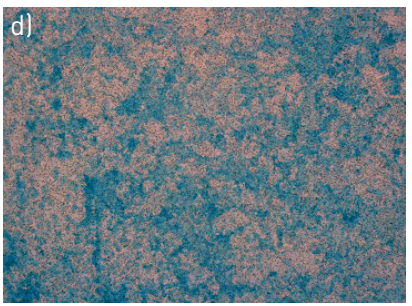

$\mathrm{CCL} 2010 \mathrm{ng} \cdot \mathrm{mL}^{-1}$

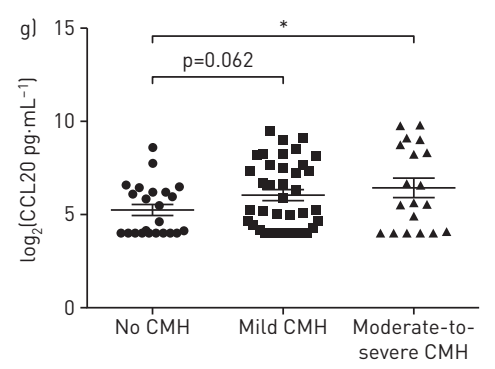

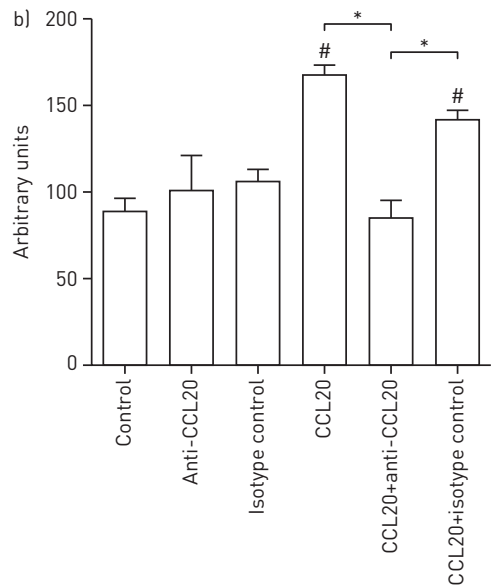

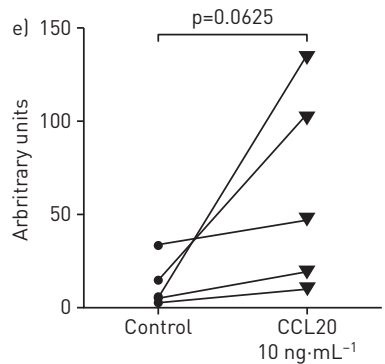

FIGURE 4 CCL20 effect on mucus production in CALU-3 and primary airway epithelial cells grown at the airliquid interface (ALI). CMH: chronic mucus hypersecretion. a) Representative images of Alcian blue staining of CALU-3 cells grown at the ALI treated on day 5 with either complete DMEM (control), rabbit anti-human CCL20 antibody, isotype control, $10 \mathrm{ng} \cdot \mathrm{mL}^{-1} \mathrm{CCL} 20$, rabbit anti-human CCL20 antibody+10 $\mathrm{ng} \cdot \mathrm{mL}^{-1} \mathrm{CCL} 20$ and isotype control+10 ng.mL ${ }^{-1}$ CCL20 for $48 \mathrm{~h}(\mathrm{n}=3)$ for each. b) Densitometry analysis of Alcian blue staining in CALU-3 cells. c-e) Representative images of Alcian blue staining of primary airway epithelial cells grown at the ALI treated on day 28 with either c) PBS or d) $10 \mathrm{ng} \cdot \mathrm{mL}^{-1}$ CCL20 for $48 \mathrm{~h}$ and e) densitometry analysis $(n=5)$. f) MUC5AC protein measurement from ALI washes ( $=5)$. g) CCL20 levels in sputum from patients with no $\mathrm{CMH}$, mild $\mathrm{CMH}$ or moderate-to-severe $\mathrm{CMH}$. Data are presented as mean \pm SEM. Statistical analysis was

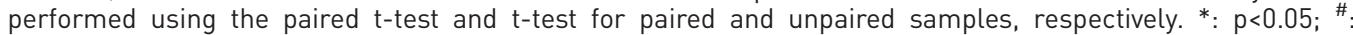
$p<0.05$, compared with control. A Wilcoxon analysis was conducted on matched samples of primary airway epithelial cells grown at the ALI. 
$\mathrm{CMH}$ and 19 were classified as having moderate-to-severe CMH. CCL20 protein levels in sputum were found to be significantly increased when comparing moderate-to-severe $\mathrm{CMH}$ with no $\mathrm{CMH}(\mathrm{p}<0.05)$, and a trend towards an increase was observed between mild and no $\mathrm{CMH}(\mathrm{p}=0.062)$ (figure $4 \mathrm{~g})$, further supporting the role of CCL20 in mucus production. As smoking may be a confounding factor, this analysis was repeated in nonsmoking patients only, where CCL20 sputum levels were also significantly increased in moderate-to-severe CMH $(\mathrm{n}=11)$ compared with no $\mathrm{CMH}(\mathrm{n}=21)$.

Our current findings indicate that IL-1 $\beta$ produced by the airway epithelium following insult induces CCL20 production by the airway smooth muscle mass that is increased in asthmatic ASMCs. This CCL20 can then act on the airway epithelium by binding to CCR6, resulting in increased mucus production. CCL20 production is inhibited by miR-146a-5p expression, which is induced by NF- $\kappa \mathrm{B}$ activation. However, this miR-146a-5p induction is lower in asthmatic ASMCs (figure 5)

\section{Discussion}

In this study we compared gene expression profiles between asthmatic and healthy ASMCs in vitro in response to the NLRP3 inflammasome downstream mediator IL-1 $\beta$. Through this analysis, we provide genome-wide evidence that ASMCs respond to the active form of IL-1 $\beta$, levels of which are increased in the sputum of asthmatic patients [7], providing a source of inflammatory chemokines and cytokines in the airways. Furthermore, we observed enhanced expression of CCL20 and MIR146A in response to IL-1 $\beta$ in ASMCs, with a lower increase in MIR146A in asthmatic ASMCs. Furthermore, IL-1 $\beta$ induced a stronger increase in CCL20 protein secretion by ASMCs from moderate compared with mild asthmatic patients and healthy controls. Interestingly, CCL20 release was reduced following overexpression of miR-146a-5p, providing evidence that this miRNA may be a dysregulated inhibitor of CCL20 production in ASMCs from asthmatic patients. Recombinant CCL20 directly induced mucus production from differentiated airway epithelial cells, indicating that CCL20 may contribute to CMH in asthma. The importance of this finding was corroborated by our observation that CCL20 levels in sputum were associated with increased levels of $\mathrm{CMH}$ in asthmatic patients. The current study reinforces the hypothesis that airway smooth muscle is not a passive bystander in inflammation and $\mathrm{CMH}$, but a key driver [30, 31].

miR-146a-5p has previously been found to regulate CCL20 production in skin keratinocytes after Toll-like receptor 2 stimulation, mirroring the results in this study [27]. However, this repression of inflammatory cytokines is not limited to CCL20, as the overexpression of miR-146a-5p led to the decrease of well-known NF- $\kappa$ B-regulated pro-inflammatory cytokines UBD, CXCL10 and CXCL8. Previous studies have identified miR-146a-5p as an anti-inflammatory miRNA that inhibits NF- $\kappa \mathrm{B}$ signalling by targeting the IL-1R downstream signalling molecules IL-1 receptor-associated kinase 1 and tumour necrosis factor

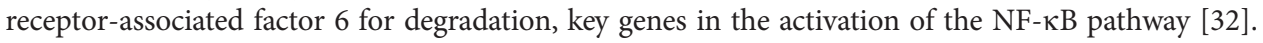

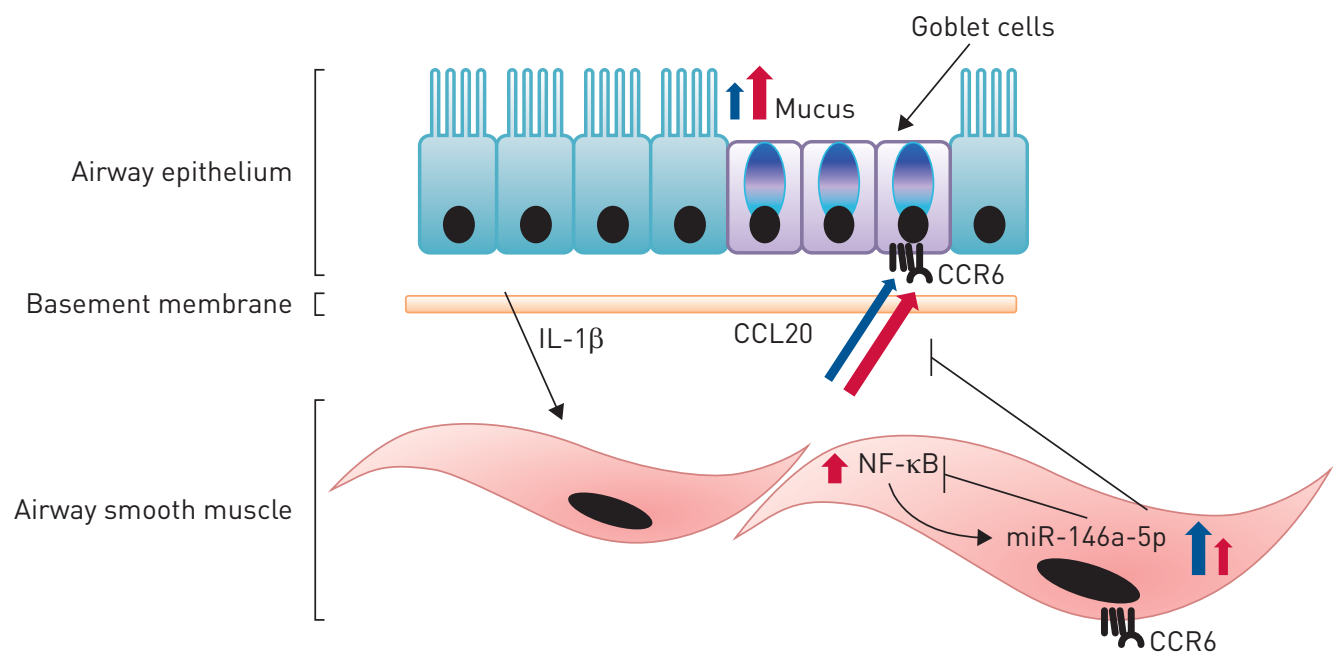

FIGURE 5 Summary of the cross-talk between the airway epithelium and airway smooth muscle cells (ASMCs) in the asthmatic airway. IL: interleukin. Basolaterally secreted IL-1 $\beta$ produced by the damaged airway epithelium induces CCL20 production by ASMCs, which is increased in asthmatic ASMCs. CCL20 can subsequently act on the airway epithelium by binding to its only known receptor CCR6, resulting in increased mucus production. CCL20 production is inhibited by miR-146a-5p expression, which is induced upon NF- $\kappa \mathrm{B}$ activation. The miR-146a-5p induction is lower in asthmatic ASMCs, leading to reduced suppression of CCL20. Red arrows: asthma; blue arrows: healthy 
In this study we found that induction of MIR146A in response to IL-1 $\beta$ was lower in asthmatic ASMCs compared with healthy controls, which may thus be responsible for the increased secretion of CCL20 from these cells. Notably, we validated the lower MIR146A expression in asthmatic patients using bronchial biopsies from asthmatic patients and healthy controls. Similar findings have been reported in human inflammatory cells, where circulating $\mathrm{CD} 4^{+}$and $\mathrm{CD} 8^{+} \mathrm{T}$-cells of severe asthmatic patients expressed less miR-146a-5p than healthy controls [33]. A likely rationale for the decrease of MIR146A in asthmatic patients may be the presence of the SNP (rs2910164), which is known to influence the levels of both the pre- and mature MIR146A transcripts [34]. This SNP has previously been associated with the presence of asthma and other pro-inflammatory diseases [11, 12]. Interestingly, we have recently shown that lower expression of miR-146a-5p in bronchial biopsies is inversely correlated with $\mathrm{CMH}$ in COPD [13], highlighting miR-146a-5p as a consistent regulator of mucus regulation in respiratory diseases.

Although we observed increased CCL20 secretion from ASMCs derived from asthma patients, we did not find a significant difference in CCL20 gene expression between asthmatic and healthy controls. Furthermore, we observed a decrease in CCL20 gene expression following $72 \mathrm{~h}$ miR-146a-5p overexpression. We postulate that only once the IL- $1 \beta$-induced increase in miR-146a-5p starts to repress CCL20 expression does this lead to differences between the asthma and control groups, with an insufficient level of miR-146a-5p in asthma-derived ASMCs. The selected time-point of $8 \mathrm{~h}$ used in this study may have been too early to detect differences in CCL20 gene expression levels between ASMCs from asthma patients and healthy controls due to the absence of miR-146a-5p suppression.

CCL20 was identified as a key chemokine for immature dendritic cells [35], and has recently been described as an antimicrobial protein [36] and regulator of mucus production [9, 25]. Overall, the function of CCL20 appears to be pro-inflammatory in nature and it is upregulated in the sputum in a number of inflammatory respiratory diseases, including asthma [20, 37], COPD [38] and cystic fibrosis [39]. Of interest, $\mathrm{CMH}$ is a feature of all of these diseases in at least a subset of patients [40-42]. In the current study we found that CCL20 promoted mucus production from an ALI-differentiated airway epithelial cell line. Furthermore, there was a direct link between sputum CCL20 levels and mucus production in asthma patients. Of note, data from our group have shown that treatment with inhaled corticosteroids increases sputum levels of CCL20 [20], offering an explanation why current anti-inflammatory therapies are unable to revert mucus hypersecretion.

The main strength of this study is the multidisciplinary approach used to identify a novel gene target using mass screening approaches including microarray analysis followed by the functional interrogation of the candidate using in vitro models. There are some limitations to this study, as we were unable to determine the origin of the CCL20 levels in the sputum of the asthmatic patients. A number of cell types in addition to ASMCs, including airway epithelial cells, produce this chemokine [43]. Despite this, the increased sensitivity and size of the muscle mass in asthmatic airways provides a potential reservoir of CCL20. Furthermore, due to remodelling of the airways, the airway smooth muscle mass is in closer proximity to the epithelial layer in asthma, which may increase the cross-talk between epithelial cells and ASMCs. The measurement of MIR146A in the bronchial biopsies is reflective of its expression within a mixed population of cells in the asthmatic airways rather than a reflection of expression in the airway smooth muscle alone. Finally, our finding that recombinant CCL20 increases mucin expression does not directly prove that airway smooth muscle-derived CCL20 drives epithelial mucus production in vivo. In future studies we will use epithelium and smooth muscle cocultures to further support our current findings.

In conclusion, in this study we identified a novel pathway leading to mucus production in asthma, where increased CCL20 released from the enhanced airway smooth muscle mass may contribute to the exaggerated mucus production by airway epithelium in asthmatic airways, due to reduced suppression by miR-146a-5p.

Author contributions: A. Faiz participated in project design, microarray analysis, in vitro cellular work, writing and proofreading of the manuscript. C.J. Vermeulen, C-J. Xu and M. Van den Berge participated in miRNA analysis, writing and proofreading of the manuscript. N.H.T. ten Hacken, G.G. King, C.S. Farah, A.J. Halayko, T.H. Lee and J.P.T. Ward provided either immortalised ASMC samples or sputum samples and participated in the proofreading of the manuscript. G. Tjin helped with immunohistochemical analysis. J.K. Burgess, M. Weckmann, J.L. Black and B.G. Oliver participated in project design, providing funding for the project, writing and proofreading of the manuscript.

Conflict of interest: M. Van den Berge reports research grants (paid to university) from Teva, Chiesi and GlaxoSmithKline, outside the submitted work. J.L. Black reports grants from the National Health and Medical Research Council, during the conduct of the study. J.K. Burgess reports grants from the National Health and Medical Research Council, during the conduct of the study. 
Support statement: This work was supported by the National Health and Medical Research Council (NHMRC), Australia (grant 570867). A. Faiz was supported by an Australian Postgraduate Award and a Longfonds Junior Investigators grant (4.2.16.132JO). J.K. Burgess was supported by a NHMRC Career Development Fellowship (1032695) and a Rosalind Franklin Fellowship funded by the University of Groningen and the European Union. J.L. Black was supported by a NHMRC senior Principal Research Fellowship (571098). B.G. Oliver was supported by a NHMRC Career Development Fellowship (1026880).

\section{References}

1 Gershon AS, Guan J, Wang C, et al. Trends in asthma prevalence and incidence in Ontario, Canada, 1996-2005: a population study. Am J Epidemiol 2010; 72: 728-736.

2 Lange P, Nyboe J, Appleyard M, et al. Relation of ventilatory impairment and of chronic mucus hypersecretion to mortality from obstructive lung disease and from all causes. Thorax 1990; 45: 579-585.

3 Whitsett JA, Alenghat T. Respiratory epithelial cells orchestrate pulmonary innate immunity. Nat Immunol 2015. 16: $27-35$.

4 Bossé Y, Paré PD, Seow CY. Airway wall remodeling in asthma: from the epithelial layer to the adventitia. Curr Allergy Asthma Rep 2008; 8: 357-366.

5 Brightling CE, Ammit AJ, Kaur D, et al. The CXCL10/CXCR3 axis mediates human lung mast cell migration to asthmatic airway smooth muscle. Am J Respir Crit Care Med 2005; 171: 1103-1108.

6 Latz E, Xiao TS, Stutz A. Activation and regulation of the inflammasomes. Nat Rev Immunol 2013; 13: 397-411.

7 Simpson JL, Phipps S, Baines KJ, et al. Elevated expression of the NLRP3 inflammasome in neutrophilic asthma. Eur Respir J 2014; 43: 1067-1076.

8 Ordonez CL, Shaughnessy TE, Matthay MA, et al. Increased neutrophil numbers and IL-8 levels in airway secretions in acute severe asthma: clinical and biologic significance. Am J Respir Crit Care Med 2000; 161: $1185-1190$.

9 Kim S, Lewis C, Nadel JA. CCL20/CCR6 feedback exaggerates epidermal growth factor receptor-dependent MUC5AC mucin production in human airway epithelial (NCI-H292) cells. J Immunol 2011; 186: 3392-3400.

10 Kallal LE, Schaller MA, Lindell DM, et al. CCL20/CCR6 blockade enhances immunity to RSV by impairing recruitment of DC. Eur I Immunol 2010; 40: 1042-1052.

11 Jiménez-Morales S, Gamboa-Becerra R, Baca V, et al. MiR-146a polymorphism is associated with asthma but not with systemic lupus erythematosus and juvenile rheumatoid arthritis in Mexican patients. Tissue Antigens 2012; 80: $317-321$.

12 Hung P-S, Chang K-W, Kao S-Y, et al. Association between the rs2910164 polymorphism in pre-mir-146a and oral carcinoma progression. Oral Oncol 2012; 48: 404-408.

13 Tasena H, Van Den Berge M, Faiz A, et al. Identification of microRNAs that potentially regulate chronic mucus hypersecretion in COPD. Am Thorac Soc 2016; 193: A4047.

14 Faiz A, Tjin G, Harkness L, et al. The expression and activity of cathepsins D, H and K in asthmatic airways. PLoS One 2013; 8: e57245.

15 Faiz A, Donovan C, Nieuwenhuis MA, et al. Latrophilin receptors: novel bronchodilator targets in asthma. Thorax 2017; 72: 74-82.

16 Sukkar MB, Xie S, Khorasani NM, et al. Toll-like receptor 2, 3, and 4 expression and function in human airway smooth muscle. J Allergy Clin Immunol 2006; 118: 641-648.

17 Boudewijn IM, Postma DS, Telenga ED, et al. Effects of ageing and smoking on pulmonary computed tomography scans using parametric response mapping. Eur Respir J 2015; 46: 1193-1196.

18 Broekema M, Volbeda F, Timens W, et al. Airway eosinophilia in remission and progression of asthma: accumulation with a fast decline of $\mathrm{FEV}_{1}$. Respir Med 2010; 104: 1254-1262.

19 Broekema M, Timens W, Vonk JM, et al. Persisting remodeling and less airway wall eosinophil activation in complete remission of asthma. Am J Respir Crit Care Med 2011; 183: 310-316.

20 Zijlstra GJ, Fattahi F, Rozeveld D, et al. Glucocorticoids induce the production of the chemoattractant CCL20 in airway epithelium. Eur Respir J 2014; 44: 361-370.

21 Van Den Berge M, Kerstjens HA, Meijer RJ, et al. Corticosteroid-induced improvement in the $\mathrm{PC}_{20}$ of adenosine monophosphate is more closely associated with reduction in airway inflammation than improvement in the $\mathrm{PC}_{20}$ of methacholine. Am J Respir Crit Care Med 2001; 164: 1127-1132.

22 Van der Molen T, Willemse BW, Schokker S, et al. Development, validity and responsiveness of the Clinical COPD Questionnaire. Health Qual Life Outcomes 2003; 1: 13.

23 Mukaida N. Pathophysiological roles of interleukin-8/CXCL8 in pulmonary diseases. Am J Physiol Lung Cell Mol Physiol 2003; 284: L566-L577.

24 Alrashdan YA, Alkhouri $\mathrm{H}$, Chen $\mathrm{E}$, et al. Asthmatic airway smooth muscle CXCL10 production: mitogen-activated protein kinase JNK involvement. Am J Physiol Lung Cell Mol Physiol 2012; 302: L1118-L1127.

25 Hirota T, Takahashi A, Kubo M, et al. Genome-wide association study identifies three new susceptibility loci for adult asthma in the Japanese population. Nat Genet 2011; 43: 893-896.

26 Perry MM, Williams AE, Tsitsiou E, et al. Divergent intracellular pathways regulate interleukin-1 $\beta$-induced miR-146a and miR-146b expression and chemokine release in human alveolar epithelial cells. FEBS Lett 2009; 583: 3349-3355.

27 Meisgen F, Landén NX, Wang A, et al. MiR-146a negatively regulates TLR2-induced inflammatory responses in keratinocytes. J Invest Dermatol 2014; 134: 1931-1940.

28 Burgess JK, Boustany S, Moir LM, et al. Reduction of tumstatin in asthmatic airways contributes to angiogenesis, inflammation, and hyperresponsiveness. Am J Respir Crit Care Med 2010; 181: 106-115.

29 Hackett T-L, Singhera GK, Shaheen F, et al. Intrinsic phenotypic differences of asthmatic epithelium and its inflammatory responses to respiratory syncytial virus and air pollution. Am J Respir Cell Mol Biol 2011; 45: 1090-1100.

30 Damera G, Tliba O, Panettieri RA. Airway smooth muscle as an immunomodulatory cell. Pulm Pharmacol Ther 2009; 22: 353-359.

31 Faiz A, Burgess JK. How can microarrays unlock asthma? J Allergy 2012; 2012: 241314. 
32 Taganov KD, Boldin MP, Chang K-J, et al. NF- KB-dependent induction of microRNA miR-146, an inhibitor targeted to signaling proteins of innate immune responses. Proc Natl Acad Sci USA 2006; 103: 12481-12486.

33 Tsitsiou E, Williams AE, Moschos SA, et al. Transcriptome analysis shows activation of circulating $\mathrm{CD}^{+}{ }^{+} \mathrm{T}$ cells in patients with severe asthma. J Allergy Clin Immunol 2012; 129: 95-103.

34 Jazdzewski K, Murray EL, Franssila K, et al. Common SNP in pre-miR-146a decreases mature miR expression and predisposes to papillary thyroid carcinoma. Proc Natl Acad Sci USA 2008; 105: 7269-7274.

35 Schutyser E, Struyf S, Van Damme J. The CC chemokine CCL20 and its receptor CCR6. Cytokine Growth Factor Rev 2003; 14: 409-426.

36 Yang D, Chen Q, Hoover DM, et al. Many chemokines including CCL20/MIP-3 $\alpha$ display antimicrobial activity. J Leukoc Biol 2003; 74: 448-455.

37 Pichavant M, Charbonnier A-S, Taront S, et al. Asthmatic bronchial epithelium activated by the proteolytic allergen Der p 1 increases selective dendritic cell recruitment. J Allergy Clin Immunol 2005; 115: 771-778.

38 Demedts IK, Bracke KR, Van Pottelberge G, et al. Accumulation of dendritic cells and increased CCL20 levels in the airways of patients with chronic obstructive pulmonary disease. Am J Respir Crit Care Med 2007; 175: 998-1005.

39 Starner TD, Barker CK, Jia HP, et al. CCL20 is an inducible product of human airway epithelia with innate immune properties. Am J Respir Cell Mol Biol 2003; 29: 627-633.

40 Prescott E, Lange P, Vestbo J. Chronic mucus hypersecretion in COPD and death from pulmonary infection. Eur Respir J 1995; 8: 1333-1338.

41 Rogers DF. Airway mucus hypersecretion in asthma: an undervalued pathology? Curr Opin Pharmacol 2004; 4: 241-250.

42 Rubin BK. Mucus structure and properties in cystic fibrosis. Paediatr Respir Rev 2007; 8: 4-7.

43 Reibman J, Hsu Y, Chen LC, et al. Airway epithelial cells release MIP-3 $\alpha / C C L 20$ in response to cytokines and ambient particulate matter. Am J Respir Cell Mol Biol 2003; 28: 648-654. 